\title{
Existence of Solutions for Degenerate Elliptic Problems in Weighted Sobolev Space
}

\author{
Lili Dai, ${ }^{1,2}$ Wenjie Gao, ${ }^{1}$ and Zhongqing $\mathrm{Li}^{3}$ \\ ${ }^{1}$ School of Mathematics, Jilin University, Changchun 130012, China \\ ${ }^{2}$ Department of Mathematics, Tonghua Normal University, Tonghua 134000, China \\ ${ }^{3}$ Department of Mathematics, Jilin Normal University, Siping 136000, China
}

Correspondence should be addressed to Lili Dai; drx820115@126.com

Received 8 September 2015; Accepted 8 November 2015

Academic Editor: Nguyen C. Phuc

Copyright (c) 2015 Lili Dai et al. This is an open access article distributed under the Creative Commons Attribution License, which permits unrestricted use, distribution, and reproduction in any medium, provided the original work is properly cited.

This paper is devoted to the study of the existence of solutions to a general elliptic problem $A u+g(x, u, \nabla u)=f-\operatorname{div} F$, with $f \in L^{1}(\Omega)$ and $F \in \prod_{i=1}^{N} L^{p^{\prime}}\left(\Omega, \omega_{i}^{*}\right)$, where $A$ is a Leray-Lions operator from a weighted Sobolev space into its dual and $g(x, s, \xi)$ is a nonlinear term satisfying $g(x, s, \xi) \operatorname{sgn}(s) \geq \rho \sum_{i=1}^{N} \omega_{i}\left|\xi_{i}\right|^{p},|s| \geq h>0$, and a growth condition with respect to $\xi$. Here, $\omega_{i}, \omega_{i}^{*}$ are weight functions that will be defined in the Preliminaries.

\section{Introduction}

Let $\Omega$ be a bounded domain in $\mathbb{R}^{N}(N \geq 1)$ and let $p$ be a real number with $1<p<\infty$. Denote by $X$ the weighted Sobolev space $W_{0}^{1, p}(\Omega, \omega)$, associated with a vector of weight functions $\omega=\left\{\omega_{i}(x)\right\}_{0 \leq i \leq N}$, which is endowed with the usual norm $\|\cdot\|_{1, p, \omega}$. In this paper, we consider a general class of degenerate elliptic problems:

$$
\begin{array}{rlrl}
A u+g(x, u, \nabla u) & =\mu & & \text { in } \Omega, \\
u=0 & & \text { on } \partial \Omega,
\end{array}
$$

where $A u=-\operatorname{div}(a(x, u, \nabla u))$ and the right-hand side term $\mu=f-\operatorname{div} F$, where $f \in L^{1}(\Omega), F \in \prod_{i=1}^{N} L^{p^{\prime}}\left(\Omega, \omega_{i}^{*}\right)$. We also assume the following:

(H1) The expression

$$
\||u|\|_{X}:=\left(\sum_{i=1}^{N} \int_{\Omega}\left|\frac{\partial u(x)}{\partial x_{i}}\right|^{p} \omega_{i}(x) d x\right)^{1 / p}
$$

is a norm defined on $X$ and it is equivalent to $\|\cdot\|_{1, p, \omega}$.
(H2) There exist a weight function $\sigma(x)$ and a parameter $q$, $1<q<\infty$, such that

$$
\sigma^{1-q^{\prime}} \in L^{1}(\Omega),
$$

with $q^{\prime}=q /(q-1)$. The Hardy inequality

$$
\begin{aligned}
& \left(\int_{\Omega}|u(x)|^{q} \sigma d x\right)^{1 / q} \\
& \quad \leq C\left(\sum_{i=1}^{N} \int_{\Omega}\left|\frac{\partial u(x)}{\partial x_{i}}\right|^{p} \omega_{i}(x) d x\right)^{1 / p}
\end{aligned}
$$

holds for every $u \in X$ with a constant $C>0$ independent of $u$. Moreover, the embedding

$$
X \hookrightarrow L^{q}(\Omega, \sigma)
$$

is compact. Interested reader may refer to [1] for some examples of weights which satisfy the above Hardy inequality (see (4)). 
(H3) $a(x, s, \xi)=\left\{a_{i}(x, s, \xi)\right\}_{1 \leq i \leq N}: \Omega \times \mathbb{R} \times \mathbb{R}^{N} \rightarrow \mathbb{R}^{N}$ is a Carathéodory vector-valued function, and for all $i=1, \ldots, N$, there hold

$$
\begin{aligned}
& \left|a_{i}(x, s, \xi)\right| \leq c_{1} \omega_{i}^{1 / p}(x) \\
& \quad\left[k(x)+\sigma^{1 / p^{\prime}}|s|^{q / p^{\prime}}+\sum_{j=1}^{N} \omega_{j}^{1 / p^{\prime}}(x)\left|\xi_{j}\right|^{p-1}\right],
\end{aligned}
$$

$$
a(x, s, \xi) \cdot \xi \geq c_{0} \sum_{i=1}^{N} \omega_{i}(x)\left|\xi_{i}\right|^{p}
$$

$$
[a(x, s, \xi)-a(x, s, \eta)] \cdot(\xi-\eta)>0, \quad \xi \neq \eta \in \mathbb{R}^{N}
$$

where $k(x)$ is a positive function in $L^{p^{\prime}}(\Omega), 1 / p+$ $1 / p^{\prime}=1$, and the constants $c_{0}, c_{1}$ are both positive.

(H4) Let $g(x, s, \xi)$ be a Carathéodory function satisfying the following assumptions:

$$
g(x, s, \xi) \operatorname{sgn}(s) \geq \rho \sum_{i=1}^{N} \omega_{i}\left|\xi_{i}\right|^{p}, \quad|s| \geq h,
$$

for some $h, \rho>0$, and

$$
|g(x, s, \xi)| \leq b(|s|)\left(\sum_{i=1}^{N} \omega_{i}\left|\xi_{i}\right|^{p}+d(x)\right)
$$

with $b: \mathbb{R}^{+} \rightarrow \mathbb{R}^{+}$, a continuous increasing function, and $d(x)$, a nonnegative function in $L^{1}(\Omega)$.

In the past decade, much attention has been devoted to nonlinear elliptic equations because of their wide application to physical models such as non-Newtonian fluids, boundary layer phenomena for viscous fluids, and chemical heterogenous model. When $-\operatorname{div} F=0$, Akdim et al. [2] proved in the variational setting, under assumptions (H1)-(H4), that, for every $f \in W^{-1, p^{\prime}}\left(\Omega, \omega^{*}\right)$, with $g$ satisfying the sign condition

$$
g(x, s, \xi) \operatorname{sgn}(s) \geq 0 .
$$

Problem (1) has a solution $u \in W_{0}^{1, p}(\Omega, \omega)$, where the authors used the approach based on the strong convergence of the positive part $u_{n}^{+}$(negative part $u_{n}^{-}$) of $u_{n}$ (the approximating sequence of $u$ ). Ammar [3] extended this existence result to problems with general data $f \in L^{1}(\Omega)$, under hypotheses (H1)-(H4). They also used a similar approach to prove the existence of renormalized solutions. When $-\operatorname{div} F \neq 0$, Aharouch et al. [4] proved the existence result for problem (1), by assuming the sign condition (11). For more details on weighted Sobolev spaces, the readers may refer to [5].

Boccardo et al. [6] considered the nonlinear boundary value problem

$$
A u+g(x, u, \nabla u)=\mu
$$

where $\mu \in L^{1}(\Omega)+W^{-1, p^{\prime}}(\Omega)$ and $g(x, u, \nabla u) \in L^{1}(\Omega)$ with sign condition (9) for large values of $s$. By combining the truncation technique with some delicate test functions, the authors showed that the problem has a solution $u \in$ $W_{0}^{1, p}(\Omega)$. Mainly motivated by $[4,6]$, we investigate the elliptic problem (1) in weighted Sobolev space. By choosing test functions different from those employed in $[4,6]$, we show that problem (1) admits at least one weak solution with (9) instead of the sign condition (11). It is worth pointing out that (9) gives a sign condition on $g(x, s, \xi)$ only for large values of $s$, which brings about many difficulties. The essential one of those is that we have to construct some new test functions to obtain the a priori estimates of the approximation solutions $u_{n}$ since the usual one $T_{k}\left(u_{n}\right)$ is not a proper test function for our problem. The outline of this paper is as follows. In Section 2, we give some preliminaries and some technical lemmas. The main results will be stated and proved in Section 3.

\section{Preliminaries}

In this section, we give some preliminaries (see [5]). Throughout this section, we assume that the vector field $a(x, s, \xi)=$ $\left\{a_{i}(x, s, \xi)\right\} 1 \leq i \leq N: \Omega \times \mathbb{R} \times \mathbb{R}^{N} \rightarrow \mathbb{R}^{N}$ satisfies assumptions (6)-(8) and $g$ satisfies (9)-(10). Let $\omega=\left\{\omega_{i}(x)\right\}_{0 \leq i \leq N}$ be a vector of measurable weight functions strictly positive a.e. in $\Omega$, such that

$$
\begin{array}{r}
\omega_{i} \in L^{1}(\Omega), \\
\omega_{i}^{-1 /(p-1)} \in L^{1}(\Omega) .
\end{array}
$$

We define the weighted space with weight $\gamma$ on $\Omega$ as

$$
L^{p}(\Omega, \gamma)=\left\{u=u(x): u \gamma^{1 / p} \in L^{p}(\Omega)\right\}
$$

With this space, we equip the norm

$$
\|u\|_{p, \gamma}=\left(\int_{\Omega}|u(x)|^{p} \gamma(x) d x\right)^{1 / p} .
$$

We denote by $W_{0}^{1, p}(\Omega, \omega)$ the space of all real-valued functions $u \in L^{p}\left(\Omega, \omega_{0}\right)$ such that the derivatives (see [5]) in the sense of distributions satisfy

$$
\frac{\partial u}{\partial x_{i}} \in L^{p}\left(\Omega, \omega_{i}\right) \quad \forall i=1, \ldots, N,
$$

endowed with the norm

$$
\begin{gathered}
\|u\|_{1, p, \omega}=\left(\int_{\Omega}|u(x)|^{p} \omega_{0}(x) d x\right. \\
\left.+\sum_{i=1}^{N} \int_{\Omega}\left|\frac{\partial u(x)}{\partial x_{i}}\right|^{p} \omega_{i}(x) d x\right)^{1 / p} .
\end{gathered}
$$

Let $X:=W_{0}^{1, p}(\Omega, \omega)$ be the closure of $C_{0}^{\infty}(\Omega)$ with respect to the norm $\|\cdot\|_{1, p, \omega}$. Then, $\left(X,\|\cdot\|_{1, p, \omega}\right)$ is a reflexive Banach space whose dual is equivalent to $W^{-1, p^{\prime}}\left(\Omega, \omega^{*}\right)$, where $\omega^{*}=\left\{\omega_{i}^{*}=\right.$ $\left.\omega_{i}^{1-p^{\prime}}=\omega_{i}^{-p^{\prime} / p}\right\}, i=1, \ldots, N$, and $p^{\prime}=p /(p-1)$. As usual, for 
$s, k$ in $\mathbb{R}$, with $k \geq 0$, we denote $T_{k}(s)=\max (-k, \min (k, s))$ and $G_{k}(s)=s-T_{k}(s)$.

The following lemmas will be needed throughout this paper (refer to $[2,7]$ ).

Lemma 1. Let $a$ and $b$ be two nonnegative real numbers, and let

$$
\varphi(s)=s e^{\theta s^{2}}
$$

with $\theta=b^{2} / 4 a^{2}$. Then,

$$
a \varphi^{\prime}(s)-b|\varphi(s)| \geq \frac{a}{2}, \quad s \in \mathbb{R} .
$$

Lemma 2. Let $g \in L^{r}(\Omega, \gamma)$ and $g_{n} \in L^{r}(\Omega, \gamma)$, with $\left\|g_{n}\right\|_{L^{r}(\Omega, \gamma)} \leq c, 1<r<\infty$. If $g_{n} \rightarrow g$ a.e. in $\Omega$, then $g_{n} \rightarrow g$ weakly in $L^{r}(\Omega, \gamma)$, where $\gamma$ is a weight function on $\Omega$.

Lemma 3 (assume (H1)). Let $G: \mathbb{R} \rightarrow \mathbb{R}$ be uniformly Lipschitzian, with $G(0)=0$. Let $u \in W_{0}^{1, p}(\Omega, \omega)$. Moreover, if the set $D$ of discontinuity points of $G^{\prime}$ is finite, then

$$
\begin{aligned}
& \frac{\partial G \circ u}{x_{i}} \\
& \quad= \begin{cases}0, & \text { a.e. in }\{x \in \Omega: u(x) \in D\}, \\
G^{\prime}(u) \frac{\partial u}{\partial x_{i}}, & \text { a.e. in }\{x \in \Omega: u(x) \notin D\} .\end{cases}
\end{aligned}
$$

Lemma 4 (assume $(\mathbf{H 1})$ ). Let $u \in W_{0}^{1, p}(\Omega, \omega)$ and $T_{k}(u)$, $k \in \mathbb{R}^{+}$, be the usual truncation. Then, $T_{k}(u) \in W_{0}^{1, p}(\Omega, \omega)$. Moreover, one has

$$
T_{k}(u) \longrightarrow u \text { strongly in } W_{0}^{1, p}(\Omega, \omega) .
$$

Lemma 5 (assume (H1) and (H2)). Let $\left\{u_{n}\right\}$ be a sequence of functions in $W_{0}^{1, p}(\Omega, \omega)$ such that $u_{n} \rightarrow u$ weakly in $W_{0}^{1, p}(\Omega, \omega)$ and

$$
\begin{aligned}
& \lim _{n \rightarrow \infty} \int_{\Omega}\left[a\left(x, u_{n}, \nabla u_{n}\right)-a(x, u, \nabla u)\right] \cdot \nabla\left(u_{n}-u\right) d x \\
& \quad=0 .
\end{aligned}
$$

Then, $u_{n} \rightarrow u$ strongly in $W_{0}^{1, p}(\Omega, \omega)$.

\section{Main Results}

Firstly, we give the definition of weak solution for problem (1).

Definition 6. One says $u \in W_{0}^{1, p}(\Omega, \omega)$ is a weak solution to problem (1), provided that

$$
\begin{gathered}
\int_{\Omega} a(x, u, \nabla u) \cdot \nabla v d x+\int_{\Omega} g(x, u, \nabla u) v d x \\
=\int_{\Omega} f v d x+\int_{\Omega} F \cdot \nabla v d x
\end{gathered}
$$

Now, we will state and prove our main result on the existence of weak solutions to problem (1).

Theorem 7. Let $f$ be in $L^{1}(\Omega)$ and $F \in \prod_{i=1}^{N} L^{p^{\prime}}\left(\Omega, \omega_{i}^{*}\right)$. Then, there exists at least one solution $u$ to problem (1).

Proof. The proof will be divided into 5 steps.

Step 1 (the approximation equation). We introduce the following approximation equation of problem (1). Let $f_{n}$ be a sequence of $L^{\infty}(\Omega)$ functions that converges to $f$ strongly in $L^{1}(\Omega)$ and let $n \in N$,

$$
g_{n}(x, s, \xi)=\frac{g(x, s, \xi)}{1+(1 / n)|g(x, s, \xi)|} ;
$$

then $g_{n}(x, s, \xi)$ is bounded and satisfies (10) and

$$
g_{n}(x, s, \xi) \cdot \operatorname{sgn}(s) \geq 0,
$$

for almost every $x$ in $\Omega$, for every $\xi$ in $\mathbb{R}^{N}$, and for every $s$ in $\mathbb{R}$ with $|s| \geq h$. By the results of [2], there exists a solution $u_{n} \in W_{0}^{1, p}(\Omega, \omega)$ of

$$
\begin{aligned}
A\left(u_{n}\right)+g_{n}\left(x, u_{n}, \nabla u_{n}\right) & =f_{n}-\operatorname{div} F \quad \text { in } \Omega, \\
u_{n} & =0 \quad \text { on } \partial \Omega,
\end{aligned}
$$

which satisfies

$$
\begin{gathered}
\int_{\Omega} a\left(x, u_{n}, \nabla u_{n}\right) \cdot \nabla v d x+\int_{\Omega} g_{n}\left(x, u_{n}, \nabla u_{n}\right) v d x \\
=\int_{\Omega} f_{n} v d x+\int_{\Omega} F \cdot \nabla v d x,
\end{gathered}
$$

for every $v \in W_{0}^{1, p}(\Omega, \omega) \cap L^{\infty}(\Omega)$.

Step 2 (the weak convergence $u_{n} \rightarrow u$ in $W_{0}^{1, p}(\Omega, \omega)$ ). Take $v=\varphi\left(T_{h}\left(u_{n}\right)\right)$ as a test function in (27), where $h>0$ is defined in (9) and $\varphi(s)$ is as in (19). Writing $\varphi_{h}^{\prime}=\varphi^{\prime}\left(T_{h}\left(u_{n}\right)\right)$ and $\varphi_{h}=$ $\varphi\left(T_{h}\left(u_{n}\right)\right)$ for simplicity, we have

$$
\begin{aligned}
\int_{\Omega} & \left(a\left(x, u_{n}, \nabla u_{n}\right)\right) \cdot \nabla T_{h}\left(u_{n}\right) \varphi_{h}^{\prime} d x \\
& +\int_{\Omega} g_{n}\left(x, u_{n}, \nabla u_{n}\right) \varphi_{h} d x \\
= & \int_{\Omega} f_{n} \varphi_{h} d x+\int_{\Omega} F \cdot \nabla T_{h}\left(u_{n}\right) \varphi_{h}^{\prime} d x .
\end{aligned}
$$

Thanks to Young's inequality and (7), we have

$$
\begin{aligned}
c_{0} \int_{\Omega} \sum_{i=1}^{N}\left|\frac{\partial T_{h}\left(u_{n}\right)}{\partial x_{i}}\right|^{p} \omega_{i}(x) \varphi_{h}^{\prime} d x \\
\quad+\int_{\Omega} g_{n}\left(x, u_{n}, \nabla u_{n}\right) \varphi_{h} d x \\
\leq \varphi(h) \int_{\Omega}\left|f_{n}\right| d x+\varphi^{\prime}(h) \int_{\Omega} \sum_{i=1}^{N}\left|F_{i} \cdot \omega_{i}^{-1 / p}\right|^{p^{\prime}} d x \\
\quad+\frac{c_{0}}{2} \int_{\Omega} \sum_{i=1}^{N}\left|\frac{\partial T_{h}\left(u_{n}\right)}{\partial x_{i}}\right|^{p} \omega_{i}(x) \varphi_{h}^{\prime} d x .
\end{aligned}
$$


Since $\left\{f_{n}\right\}$ is bounded in $L^{1}(\Omega)$ and $F \in \prod_{i=1}^{N} L^{p^{\prime}}\left(\Omega, \omega_{i}^{*}\right)$, it follows from the above inequality that

$$
\begin{aligned}
& \frac{c_{0}}{2} \int_{\Omega} \sum_{i=1}^{N}\left|\frac{\partial T_{h}\left(u_{n}\right)}{\partial x_{i}}\right|^{p} \omega_{i}(x) \varphi_{h}^{\prime} d x \\
& +\int_{\Omega} g_{n}\left(x, u_{n}, \nabla u_{n}\right) \varphi_{h} d x \leq C,
\end{aligned}
$$

where $C$ is independent of $n$. Splitting the second term on the left-hand side where $\left|u_{n}\right|<h$ and $\left|u_{n}\right| \geq h$, we can write

$$
\begin{aligned}
& \int_{\Omega} g_{n}\left(x, u_{n}, \nabla u_{n}\right) \varphi_{h} d x \\
& =\int_{\left\{\left|u_{n}\right|<h\right\}} g_{n}\left(x, u_{n}, \nabla u_{n}\right) \varphi_{h} d x \\
& \quad+\int_{\left\{\left|u_{n}\right| \geq h\right\}} g_{n}\left(x, u_{n}, \nabla u_{n}\right) \varphi_{h} d x .
\end{aligned}
$$

Using (9) and (10), we get

$$
\begin{gathered}
\int_{\left\{\left|u_{n}\right| \geq h\right\}} g_{n}\left(x, u_{n}, \nabla u_{n}\right) \varphi_{h} d x \geq \rho \varphi(h) \\
\cdot \int_{\left\{\left|u_{n}\right| \geq h\right\}} \sum_{i=1}^{N}\left|\frac{\partial u_{n}}{\partial x_{i}}\right|^{p} \omega_{i}(x) d x, \\
\left|\int_{\left\{\left|u_{n}\right|<h\right\}} g_{n}\left(x, u_{n}, \nabla u_{n}\right) \varphi_{h} d x\right| \leq b(h) \\
\cdot\left(\int_{\Omega} \sum_{i=1}^{N}\left|\frac{\partial T_{h}\left(u_{n}\right)}{\partial x_{i}}\right|^{p} \omega_{i}(x) \varphi_{h} d x\right. \\
\left.+\varphi(h)\|d\|_{L^{1}(\Omega)}\right) .
\end{gathered}
$$

Hence,

$$
\begin{array}{r}
\int_{\Omega} \sum_{i=1}^{N}\left|\frac{\partial T_{h}\left(u_{n}\right)}{\partial x_{i}}\right|^{p} \omega_{i}(x)\left[\frac{c_{0}}{2} \varphi_{h}^{\prime}-b(h)\left|\varphi_{h}\right|\right] d x \\
+\rho \varphi(h) \int_{\left\{\left|u_{n}\right| \geq h\right\}} \sum_{i=1}^{N}\left|\frac{\partial u_{n}}{\partial x_{i}}\right|^{p} \omega_{i}(x) d x \leq C .
\end{array}
$$

Recalling (19) in Lemma 1 , let $a=c_{0} / 2, b=b(h)$; we then obtain

$$
\begin{aligned}
& \int_{\Omega} \sum_{i=1}^{N}\left|\frac{\partial T_{h}\left(u_{n}\right)}{\partial x_{i}}\right|^{p} \omega_{i}(x) d x \\
& \quad+\int_{\left\{\left|u_{n}\right| \geq h\right\}} \sum_{i=1}^{N}\left|\frac{\partial u_{n}}{\partial x_{i}}\right|^{p} \omega_{i}(x) d x \leq C,
\end{aligned}
$$

which implies

$$
\int_{\Omega} \sum_{i=1}^{N}\left|\frac{\partial u_{n}}{\partial x_{i}}\right|^{p} \omega_{i}(x) d x \leq C,
$$

or equivalently

$$
\left\|\left|u_{n}\right|\right\|_{X} \leq C
$$

where $C$ is some positive constant. Therefore, we can extract a subsequence, still denoted by itself, such that

$$
u_{n} \rightarrow u \quad \text { weakly in } W_{0}^{1, p}(\Omega, \omega) .
$$

By (5) and (37), we have for a subsequence $u_{n} \rightarrow u$ strongly in $L^{q}(\Omega, \omega)$ and a.e. in $\Omega$. Then, $T_{k}\left(u_{n}\right)$ is bounded in $W_{0}^{1, p}(\Omega, \omega)$. Hence, by the results of [8], we have

$$
T_{k}\left(u_{n}\right) \rightarrow T_{k}(u) \quad \text { weakly in } W_{0}^{1, p}(\Omega, \omega) .
$$

Step 3 (the strong convergence $u_{n} \rightarrow u$ in $W_{0}^{1, p}(\Omega, \omega)$ ). For every $k \geq h$, we will prove that $T_{k}\left(u_{n}\right)$ converges strongly to $T_{k}(u)$ in $W_{0}^{1, p}(\Omega, \omega)$. We first prove that

$$
\lim _{k \rightarrow+\infty} \sup _{n \in N^{*}} \int_{\left\{\left|u_{n}\right| \geq k\right\}} \sum_{i=1}^{N}\left|\frac{\partial u_{n}}{\partial x_{i}}\right|^{p} \omega_{i} d x=0 .
$$

Here, we denote by $N^{*}$ the set of natural numbers. Choosing $v=T_{k}\left(u_{n}\right)-T_{k-1}\left(u_{n}\right)$ as a test function in (27) with $k \geq h+1$, using (7) and Young's inequality, we obtain

$$
\begin{aligned}
& \frac{c_{0}}{2} \int_{\Omega} \sum_{i=1}^{N} \omega_{i}(x)\left|\frac{\partial\left(T_{k}\left(u_{n}\right)-T_{k-1}\left(u_{n}\right)\right)}{\partial x_{i}}\right|^{p} d x \\
& \quad+\int_{\Omega} g_{n}\left(x, u_{n}, \nabla u_{n}\right)\left(T_{k}\left(u_{n}\right)-T_{k-1}\left(u_{n}\right)\right) d x \\
& \leq \int_{\left\{\left|u_{n}\right| \leq k-1\right\}} f_{n}\left(T_{k}\left(u_{n}\right)-T_{k-1}\left(u_{n}\right)\right) d x \\
& \quad+\int_{\left\{k-1 \leq\left|u_{n}\right| \leq k\right\}} \sum_{i=1}^{N}\left|F_{i} \cdot \omega_{i}^{-1 / p}\right|^{p^{\prime}} d x .
\end{aligned}
$$

Noticing (9) and that $T_{k}\left(u_{n}\right)-T_{k-1}\left(u_{n}\right)$ has the same sign as $g_{n}\left(x, u_{n}, \nabla u_{n}\right)$ if $\left|u_{n}\right|>h$ and is zero if $\left|u_{n}\right| \leq h$, we get

$$
\begin{aligned}
& g_{n}\left(x, u_{n}, \nabla u_{n}\right)\left(T_{k}\left(u_{n}\right)-T_{k-1}\left(u_{n}\right)\right) \\
& \quad \geq\left|g_{n}\left(x, u_{n}, \nabla u_{n}\right)\right| \chi_{\left\{\left|u_{n}\right| \geq k\right\} .} .
\end{aligned}
$$

Dropping the nonnegative term, we have

$$
\begin{aligned}
& \int_{\left\{\left|u_{n}\right| \geq k\right\}}\left|g_{n}\left(x, u_{n}, \nabla u_{n}\right)\right| d x \\
& \leq \int_{\left\{\left|u_{n}\right| \geq k-1\right\}}\left|f_{n}\right| d x \\
& \quad+\int_{\left\{k-1 \leq\left|u_{n}\right| \leq k\right\}} \sum_{i=1}^{N}\left|F_{i} \cdot \omega_{i}^{-1 / p}\right|^{p^{\prime}} d x .
\end{aligned}
$$

Since

$$
\begin{aligned}
\operatorname{meas}\left\{\left|u_{n}\right| \geq k\right\} & \leq \int_{\Omega} \frac{\left|u_{n}\right| \omega_{0}^{1 / p}}{k}\left(\omega_{0}^{-1}(x)\right)^{1 / p} d x \\
& \leq \frac{1}{k}\left\|u_{n}\right\|_{L^{p}\left(\Omega, \omega_{0}\right)}\left\|\left(\omega_{0}^{-1}\right)^{1 / p}\right\|_{L^{p^{\prime}}(\Omega)} \\
& \leq \frac{1}{k}\left\|u_{n}\right\|_{L^{p}\left(\Omega, \omega_{0}\right)}\left\|\left(\omega_{0}^{-1}\right)\right\|_{L^{1 /(p-1)}(\Omega)}^{1 / p},
\end{aligned}
$$


we obtain

$$
\lim _{k \rightarrow+\infty} \sup _{n \in N^{*}} \operatorname{meas}\left(\left\{\left|u_{n}\right| \geq k-1\right\}\right)=0 .
$$

Taking into account the fact that $\left\{f_{n}\right\}$ is compact in $L^{1}(\Omega)$ and $F \in \prod_{i=1}^{N} L^{p^{\prime}}\left(\Omega, \omega_{i}^{*}\right)$, we deduce that

$$
\begin{aligned}
\lim _{k \rightarrow+\infty} & \sup _{n \in N^{*}} \int_{\left\{\left|u_{n}\right| \geq k-1\right\}}\left|f_{n}\right| d x \\
& +\int_{\left\{k-1 \leq\left|u_{n}\right| \leq k\right\}} \sum_{i=1}^{N}\left|F_{i} \cdot \omega_{i}^{-1 / p}\right|^{p^{\prime}} d x=0 .
\end{aligned}
$$

Hence,

$$
\lim _{k \rightarrow+\infty} \sup _{n \in N^{*}} \int_{\left\{\left|u_{n}\right| \geq k\right\}}\left|g_{n}\left(x, u_{n}, \nabla u_{n}\right)\right| d x=0 .
$$

Noticing that $k \geq h$ and (9), this completes the proof of assertion (39).

Let $k \geq h$ be fixed, $0<\varepsilon<k$, and choose $v=\varphi\left(T_{\varepsilon}\left(u_{n}-\right.\right.$ $\left.\left.T_{k}(u)\right)\right)$ as a test function in (27), where $\varphi(s)$ is defined in Lemma 1 (refer to [8-10]). We thus obtain

$$
\begin{aligned}
& \underbrace{\int_{\Omega} a\left(x, u_{n}, \nabla u_{n}\right) \cdot \nabla\left(T_{\varepsilon}\left(u_{n}-T_{k}(u)\right)\right) \varphi^{\prime}\left(T_{\varepsilon}\left(u_{n}-T_{k}(u)\right)\right) d x}_{\Omega} \\
& +\underbrace{\int_{\Omega} g_{n}\left(x, u_{n}, \nabla u_{n}\right) \varphi\left(T_{\varepsilon}\left(u_{n}-T_{k}(u)\right)\right) d x}_{(A)} \\
& =\underbrace{\int_{\Omega} f_{n} \varphi\left(T_{\varepsilon}\left(u_{n}-T_{k}(u)\right)\right) d x}_{(B)} \\
& \quad+\underbrace{\int_{\Omega} F \cdot \nabla\left(T_{\varepsilon}\left(u_{n}-T_{k}(u)\right)\right) \varphi^{\prime}\left(T_{\varepsilon}\left(u_{n}-T_{k}(u)\right)\right) d x .}_{(D)}
\end{aligned}
$$

$$
\begin{aligned}
& \underbrace{\int_{\Omega} a\left(x, T_{k+\varepsilon}\left(u_{n}\right), \nabla T_{k+\varepsilon}\left(u_{n}\right)\right) \cdot \nabla\left(T_{\varepsilon}\left(u_{n}-T_{k}(u)\right)\right) \varphi_{\varepsilon, n}^{\prime} d x}_{\Omega} \\
& +\underbrace{\int_{\Omega} a\left(x, u_{n}, \nabla G_{k+\varepsilon}\left(u_{n}\right)\right) \chi\left\{\left|u_{n}\right| \geq k+\varepsilon\right\} \nabla\left(T_{\varepsilon}\left(u_{n}-T_{k}(u)\right)\right) \varphi_{\varepsilon, n}^{\prime} d x}_{(F)} .
\end{aligned}
$$

Owing to $\left\{\left|u_{n}-T_{k}(u)\right| \leq \varepsilon\right\} \subset\left\{\left|u_{n}\right| \leq k+\varepsilon\right\}$, we get

$$
\begin{aligned}
& (F)=\int_{\Omega} a\left(x, u_{n}, \nabla G_{k+\varepsilon}\left(u_{n}\right)\right) \chi\left\{\left|u_{n}\right| \geq k+\varepsilon\right\} \\
& \quad \cdot \nabla\left(T_{k+\varepsilon}\left(u_{n}\right)-T_{k}(u)\right) \chi_{\varepsilon}\left\{\left|u_{n}-T_{k}(u)\right|\right\} \varphi_{\varepsilon, n}^{\prime} d x .
\end{aligned}
$$

Since $\nabla T_{k+\varepsilon}\left(u_{n}\right)$ is zero whenever $\nabla G_{k+\varepsilon}\left(u_{n}\right)$ is not zero, hence,

$$
\begin{aligned}
& (F)=-\int_{\Omega} a\left(x, u_{n}, \nabla G_{k+\varepsilon}\left(u_{n}\right)\right) \\
& \cdot \nabla T_{k}(u) \chi\left\{\left|u_{n}\right|>k+\varepsilon\right\} \chi_{\varepsilon}\left\{\left|u_{n}-T_{k}(u)\right|\right\} \varphi_{\varepsilon, n}^{\prime} d x .
\end{aligned}
$$

In the following, $\delta(\varepsilon, n)$ represents a quantity which converges to zero as firstly $n \rightarrow \infty$ and secondly $\varepsilon \rightarrow 0$. For convenience, we write

$$
\begin{aligned}
& \varphi_{\varepsilon, n}^{\prime}=\varphi^{\prime}\left(T_{\varepsilon}\left(u_{n}-T_{k}(u)\right)\right), \\
& \varphi_{\varepsilon, n}=\varphi\left(T_{\varepsilon}\left(u_{n}-T_{k}(u)\right)\right) .
\end{aligned}
$$

Observe that, in the weak ${ }^{*}$ topology of $L^{\infty}(\Omega)$ and almost everywhere in $\Omega$, we have

$$
\begin{aligned}
& \lim _{\varepsilon \rightarrow 0} \lim _{n \rightarrow \infty} \varphi_{\varepsilon, n}=0, \\
& \lim _{\varepsilon \rightarrow 0} \lim _{n \rightarrow \infty} \varphi_{\varepsilon, n}^{\prime}=1 .
\end{aligned}
$$

Now, as $\left\{f_{n}\right\}$ is compact in $L^{1}(\Omega)$ and (49), we have

$$
(C)=\delta(\varepsilon, n) \text {. }
$$

Thanks to $T_{k+\varepsilon}\left(u_{n}\right) \rightarrow T_{k}(u)$ weakly in $W_{0}^{1, p}(\Omega, \omega), F \in$ $\prod_{i=1}^{N} L^{p^{\prime}}\left(\Omega, \omega_{i}^{*}\right)$, and (49), we obtain

$$
\begin{aligned}
(D) & =\int_{\Omega} F \\
\cdot & \nabla\left(T_{k+\varepsilon}\left(u_{n}\right)-T_{k}(u)\right) \chi_{\varepsilon}\left\{\left|u_{n}-T_{k}(u)\right|\right\} \varphi_{\varepsilon, n}^{\prime} d x \\
= & \delta(\varepsilon, n),
\end{aligned}
$$

where $\chi_{\varepsilon}\left\{\left|u_{n}-T_{k}(u)\right|\right\}=\chi\left\{\left|u_{n}-T_{k}(u)\right| \leq \varepsilon\right\}$. We can decompose $(A)$ as
Since $\nabla T_{k}(u) \equiv 0$ on the set $\{|u|>k+\varepsilon\}$, we see that, as $n \rightarrow \infty$,

$$
\nabla T_{k}(u) \chi_{\left\{\left|u_{n}\right|>k+\varepsilon\right\}} \longrightarrow 0, \quad \text { a.e. in } \Omega \text {. }
$$

As $\nabla T_{k}(u) \in \prod_{i=1}^{N} L^{p}\left(\Omega, \omega_{i}\right)$, Lebesgue's dominated convergence theorem guarantees that

$$
\begin{aligned}
& \nabla T_{k}(u) \chi_{\left\{\left|u_{n}\right|>k+\varepsilon\right\}} \longrightarrow 0 \\
& \text { strongly in } \prod_{i=1}^{N} L^{p}\left(\Omega, \omega_{i}\right), n \longrightarrow \infty .
\end{aligned}
$$


By (6), (49), and the fact that $a\left(x, u_{n}, \nabla G_{k+\varepsilon}\left(u_{n}\right)\right)$ is bounded Now we split $(E)$ into in $\prod_{i=1}^{N} L^{p^{\prime}}\left(\Omega, \omega_{i}^{*}\right)$, we obtain

$$
(F)=\delta(\varepsilon, n) \text {. }
$$

$$
\begin{aligned}
& \underbrace{\int_{\Omega}\left[a\left(x, T_{k+\varepsilon}\left(u_{n}\right), \nabla T_{k+\varepsilon}\left(u_{n}\right)\right)-a\left(x, T_{k+\varepsilon}\left(u_{n}\right), \nabla T_{k}(u)\right)\right] \cdot \nabla\left(T_{k+\varepsilon}\left(u_{n}\right)-T_{k}(u)\right) \varphi_{\varepsilon, n}^{\prime} d x}_{(G)} \\
& +\underbrace{\int_{\Omega} a\left(x, T_{k+\varepsilon}\left(u_{n}\right), \nabla T_{k}(u)\right) \cdot \nabla\left(T_{k+\varepsilon}\left(u_{n}\right)-T_{k}(u)\right) \varphi_{\varepsilon, n}^{\prime} d x .}_{(H)}
\end{aligned}
$$

We will prove that

$$
\begin{aligned}
a_{i}\left(x, T_{k+\varepsilon}\left(u_{n}\right), \nabla T_{k}(u)\right) \longrightarrow & a_{i}\left(x, T_{k+\varepsilon}(u), \nabla T_{k}(u)\right) \\
& \text { strongly in } L^{p^{\prime}}\left(\Omega, \omega_{i}^{*}\right) .
\end{aligned}
$$

In fact,

$$
\begin{aligned}
& \left|a_{i}\left(x, T_{k+\varepsilon}\left(u_{n}\right), \nabla T_{k}(u)\right)\right|^{p^{\prime}} \omega_{i}^{-p^{\prime} / p} \leq c_{1}[k(x) \\
& +\sigma^{1 / p^{\prime}}\left|T_{k+\varepsilon}\left(u_{n}\right)\right|^{q / p^{\prime}} \\
& \left.\quad+\sum_{i=1}^{N} \omega_{i}^{1 / p^{\prime}}(x)\left|\frac{\partial T_{k}(u)}{\partial x_{i}}\right|^{p-1}\right]^{p^{\prime}} \leq c_{2}\left[k(x)^{p^{\prime}}\right. \\
& \left.\quad+\sigma\left|T_{k+\varepsilon}\left(u_{n}\right)\right|^{q}+\sum_{i=1}^{N} \omega_{i}(x)\left|\frac{\partial T_{k}(u)}{\partial x_{i}}\right|^{p}\right],
\end{aligned}
$$

where $c_{1}, c_{2}$ are positive constants. Since $T_{k+\varepsilon}\left(u_{n}\right) \rightarrow T_{k+\varepsilon}(u)$ weakly in $W_{0}^{1, p}(\Omega, \omega)$ and $W_{0}^{1, p}(\Omega, \omega) \hookrightarrow L^{q}(\Omega, \sigma)$ is compact, then $T_{k+\varepsilon}\left(u_{n}\right) \rightarrow T_{k+\varepsilon}(u)$ strongly in $L^{q}(\Omega, \sigma)$ and a.e. in $\Omega$. Hence,

$$
\begin{aligned}
& \left|a_{i}\left(x, T_{k+\varepsilon}\left(u_{n}\right), \nabla T_{k}(u)\right)\right|^{p^{\prime}} \omega_{i}^{*} \\
& \longrightarrow\left|a_{i}\left(x, T_{k+\varepsilon}(u), \nabla T_{k}(u)\right)\right|^{p^{\prime}} \omega_{i}^{*} \text { a.e. in } \Omega, \\
& c_{2}\left[k(x)^{p^{\prime}}+\sigma\left|T_{k+\varepsilon}\left(u_{n}\right)\right|^{q}+\sum_{i=1}^{N} \omega_{i}(x)\left|\frac{\partial T_{k}(u)}{\partial x_{i}}\right|^{p}\right] \\
& \longrightarrow c_{2}\left[k(x)^{p^{\prime}}+\sigma\left|T_{k+\varepsilon}(u)\right|^{q}\right. \\
& \left.\quad+\sum_{o=1}^{N} \omega_{i}(x)\left|\frac{\partial T_{k}(u)}{\partial x_{i}}\right|^{p}\right] \text { strongly in } L^{q}(\Omega, \sigma) .
\end{aligned}
$$

Then, by the generalized Lebesgue dominated convergence theorem, we deduce (59). By $\partial T_{k+\varepsilon}\left(u_{n}\right) / \partial x_{i} \rightarrow \partial T_{k}(u) / \partial x_{i}$ weakly in $L^{p}\left(\Omega, \omega_{i}\right)$ and (49), we have

$$
(H)=\delta(\varepsilon, n) \text {. }
$$

Using (57) and (62), we have

$$
\begin{aligned}
(A) & =\int_{\Omega}\left[a\left(x, T_{k+\varepsilon}\left(u_{n}\right), \nabla T_{k+\varepsilon}\left(u_{n}\right)\right)\right. \\
& \left.-a\left(x, T_{k+\varepsilon}\left(u_{n}\right), \nabla T_{k}(u)\right)\right] \cdot \nabla\left(T_{k+\varepsilon}\left(u_{n}\right)\right. \\
& \left.-T_{k}(u)\right) \varphi_{\varepsilon, n}^{\prime} d x+\delta(\varepsilon, n) .
\end{aligned}
$$

As for the term $(B)$, we decompose it as

$$
\begin{aligned}
& \underbrace{\int_{\left\{\left|u_{n}\right| \geq k+\varepsilon\right\}} g_{n}\left(x, u_{n}, \nabla u_{n}\right) \varphi_{\varepsilon, n} d x}_{(I)} \\
& +\underbrace{\int_{\left\{\left|u_{n}\right|<k+\varepsilon\right\}} g_{n}\left(x, u_{n}, \nabla u_{n}\right) \varphi_{\varepsilon, n} d x}_{(J)} .
\end{aligned}
$$

It is clear that on the set $\left\{u_{n} \geq k+\varepsilon\right\}$ we get

$$
\varphi_{\varepsilon, n}=\varphi\left(T_{\varepsilon}\left(u_{n}-T_{k}(u)\right)\right)=\varphi(\varepsilon) \geq 0,
$$

while on the set $\left\{u_{n}<-k-\varepsilon\right\}$ we get

$$
\varphi_{\varepsilon, n}=\varphi\left(T_{\varepsilon}\left(u_{n}-T_{k}(u)\right)\right)=\varphi(-\varepsilon) \leq 0 .
$$

By (9) and the fact that $k \geq h$, we obtain

$$
(I) \geq 0 \text {. }
$$

Using (7) and (10) and noticing that $\varepsilon<k$, we have

$$
\begin{aligned}
& |(J)| \leq \int_{\left\{\left|u_{n}\right| \leq k+\varepsilon\right\}} b(2 k)\left[d(x)+\sum_{i=1}^{N}\left|\frac{\partial u_{n}}{\partial x_{i}}\right|^{p} \omega_{i}(x)\right] \\
& \cdot\left|\varphi_{\varepsilon, n}\right| d x \leq b(2 k) \int_{\left\{\left|u_{n}\right| \leq k+\varepsilon\right\}} d(x)\left|\varphi_{\varepsilon, n}\right| d x \\
& +\frac{b(2 k)}{c_{0}} \int_{\left\{\left|u_{n}\right| \leq k+\varepsilon\right\}} a\left(x, u_{n}, \nabla u_{n}\right) \nabla u_{n}\left|\varphi_{\varepsilon, n}\right| d x \\
& =\delta(\varepsilon, n)+\frac{b(2 k)}{c_{0}} \int_{\Omega} a\left(x, T_{k+\varepsilon}\left(u_{n}\right), \nabla T_{k+\varepsilon}\left(u_{n}\right)\right) \\
& \cdot \nabla T_{k+\varepsilon}\left(u_{n}\right)\left|\varphi_{\varepsilon, n}\right| d x \leq \frac{b(2 k)}{c_{0}} \\
& \cdot \int_{\Omega}\left[a\left(x, T_{k+\varepsilon}\left(u_{n}\right), \nabla T_{k+\varepsilon}\left(u_{n}\right)\right)\right.
\end{aligned}
$$




$$
\begin{aligned}
& \left.-a\left(x, T_{k+\varepsilon}\left(u_{n}\right), \nabla T_{k}(u)\right)\right] \cdot \nabla\left(T_{k+\varepsilon}\left(u_{n}\right)\right. \\
& \left.-T_{k}(u)\right)\left|\varphi_{\varepsilon, n}\right| d x+\int_{\Omega} a\left(x, T_{k+\varepsilon}\left(u_{n}\right), \nabla T_{k}(u)\right) \\
& \cdot \nabla\left(T_{k+\varepsilon}\left(u_{n}\right)-T_{k}(u)\right)\left|\varphi_{\varepsilon, n}\right| d x \\
& +\int_{\Omega} a\left(x, T_{k+\varepsilon}\left(u_{n}\right), \nabla T_{k+\varepsilon}\left(u_{n}\right)\right) \cdot \nabla T_{k}(u) \\
& \cdot\left|\varphi_{\varepsilon, n}\right| d x+\delta(\varepsilon, n) .
\end{aligned}
$$

By the weak convergence of $\partial T_{k+\varepsilon}\left(u_{n}\right) / \partial x_{i} \rightarrow \partial T_{k}(u) / \partial x_{i}$ in $L^{p}\left(\Omega, \omega_{i}\right),(49)$, and (59), we have

$$
\begin{aligned}
& \int_{\Omega} a\left(x, T_{k+\varepsilon}\left(u_{n}\right), \nabla T_{k}(u)\right) \\
& \quad \cdot \nabla\left(T_{k+\varepsilon}\left(u_{n}\right)-T_{k}(u)\right)\left|\varphi_{\varepsilon, n}\right| d x=\delta(\varepsilon, n) .
\end{aligned}
$$

Since $a\left(x, T_{k+\varepsilon}\left(u_{n}\right), \nabla T_{k+\varepsilon}\left(u_{n}\right)\right)$ is bounded in $\prod_{i=1}^{N} L^{p^{\prime}}\left(\Omega, \omega_{i}^{*}\right)$ and (49),

$$
\begin{aligned}
& \int_{\Omega} a\left(x, T_{k+\varepsilon}\left(u_{n}\right), \nabla T_{k+\varepsilon}\left(u_{n}\right)\right) \cdot \nabla T_{k}(u)\left|\varphi_{\varepsilon, n}\right| d x \\
& \quad=\delta(\varepsilon, n) .
\end{aligned}
$$

We have

$$
\begin{aligned}
& |(J)| \leq \frac{b(2 k)}{c_{0}} \int_{\Omega}\left[a\left(x, T_{k+\varepsilon}\left(u_{n}\right), \nabla T_{k+\varepsilon}\left(u_{n}\right)\right)\right. \\
& \left.\quad-a\left(x, T_{k+\varepsilon}\left(u_{n}\right), \nabla T_{k}(u)\right)\right] \cdot \nabla\left(T_{k+\varepsilon}\left(u_{n}\right)\right. \\
& \left.\quad-T_{k}(u)\right)\left|\varphi_{\varepsilon, n}\right| d x+\delta(\varepsilon, n) .
\end{aligned}
$$

Invoking (63), we have

$$
\begin{aligned}
& \int_{\Omega}\left[a\left(x, T_{k+\varepsilon}\left(u_{n}\right), \nabla T_{k+\varepsilon}\left(u_{n}\right)\right)\right. \\
& \left.-a\left(x, T_{k+\varepsilon}\left(u_{n}\right), \nabla T_{k}(u)\right)\right] \cdot \nabla\left(T_{k+\varepsilon}\left(u_{n}\right)\right. \\
& \left.\quad-T_{k}(u)\right)\left[\varphi_{\varepsilon, n}^{\prime}-\frac{b(2 k)}{c_{0}}\left|\varphi_{\varepsilon, n}\right|\right] d x=\delta(\varepsilon, n) .
\end{aligned}
$$

Consequently, by (19) and letting $a=1$ and $b=b(2 k) / c_{0}$, it yields

$$
\begin{aligned}
\int_{\Omega} & {\left[a\left(x, T_{k+\varepsilon}\left(u_{n}\right), \nabla T_{k+\varepsilon}\left(u_{n}\right)\right)\right.} \\
& \left.-a\left(x, T_{k+\varepsilon}\left(u_{n}\right), \nabla T_{k}(u)\right)\right] \cdot \nabla\left(T_{k+\varepsilon}\left(u_{n}\right)\right. \\
& \left.-T_{k}(u)\right) d x=\delta(\varepsilon, n) .
\end{aligned}
$$

Since $\left\{\left|u_{n}\right| \leq k\right\} \subset\left\{\left|u_{n}\right| \leq k+\varepsilon\right\}$, we have

$$
\begin{aligned}
\int_{\Omega} & {\left[a\left(x, T_{k}\left(u_{n}\right), \nabla T_{k}\left(u_{n}\right)\right)\right.} \\
& \left.-a\left(x, T_{k}\left(u_{n}\right), \nabla T_{k}(u)\right)\right] \cdot \nabla\left(T_{k}\left(u_{n}\right)\right. \\
& \left.-T_{k}(u)\right) d x=\int_{\left\{\left|u_{n}\right| \leq k\right\}}\left[a\left(x, u_{n}, \nabla u_{n}\right)\right. \\
& \left.-a\left(x, u_{n}, \nabla T_{k}(u)\right)\right] \cdot \nabla\left(u_{n}-T_{k}(u)\right) d x \\
& \leq \int_{\left\{\left|u_{n}\right| \leq k+\varepsilon\right\}}\left[a\left(x, u_{n}, \nabla u_{n}\right)-a\left(x, u_{n}, \nabla T_{k}(u)\right)\right] \\
& -\nabla\left(u_{n}-T_{k}(u)\right) d x \\
& =\int_{\Omega}\left[a\left(x, T_{k+\varepsilon}\left(u_{n}\right), \nabla T_{k+\varepsilon}\left(u_{n}\right)\right)\right. \\
& \left.-a\left(x, T_{k+\varepsilon}\left(u_{n}\right), \nabla T_{k}(u)\right)\right] \cdot \nabla\left(T_{k+\varepsilon}\left(u_{n}\right)\right. \\
& \left.-T_{k}(u)\right) d x=\delta(\varepsilon, n) .
\end{aligned}
$$

Together with Lemma 5 and the assumptions on $a$, we obtain

$$
T_{k}\left(u_{n}\right) \longrightarrow T_{k}(u) \quad \text { strongly in } W_{0}^{1, p}(\Omega, \omega),
$$

which in turn implies

$$
\nabla u_{n} \longrightarrow \nabla u \quad \text { a.e. in } \Omega \text {. }
$$

For any measurable set $E$ of $\Omega$, we have

$$
\begin{aligned}
\int_{E} \sum_{i=1}^{N}\left|\frac{\partial u_{n}}{\partial x_{i}}\right|^{p} \omega_{i} d x= & \int_{E \cap\left\{\left|u_{n}\right|<k\right\}} \sum_{i=1}^{N}\left|\frac{\partial u_{n}}{\partial x_{i}}\right|^{p} \omega_{i} d x \\
& +\int_{E \cap\left\{\left|u_{n}\right| \geq k\right\}} \sum_{i=1}^{N}\left|\frac{\partial u_{n}}{\partial x_{i}}\right|^{p} \omega_{i} d x .
\end{aligned}
$$

Let $\varepsilon>0$. Thanks to

$$
\int_{E \cap\left\{\left|u_{n}\right| \geq k\right\}} \sum_{i=1}^{N}\left|\frac{\partial u_{n}}{\partial x_{i}}\right|^{p} \omega_{i} d x \leq \int_{\left\{\left|u_{n}\right| \geq k\right\}} \sum_{i=1}^{N}\left|\frac{\partial u_{n}}{\partial x_{i}}\right|^{p} \omega_{i} d x,
$$

by (39), there exists $k \geq h$ such that

$$
\int_{E \cap\left\{\left|u_{n}\right| \geq k\right\}} \sum_{i=1}^{N}\left|\frac{\partial u_{n}}{\partial x_{i}}\right|^{p} \omega_{i} d x \leq \frac{\varepsilon}{2}, \quad n \in N^{*} .
$$

While $k$ is fixed, we get

$$
\int_{E \cap\left\{\left|u_{n}\right|<k\right\}} \sum_{i=1}^{N}\left|\frac{\partial u_{n}}{\partial x_{i}}\right|^{p} \omega_{i} d x \leq \int_{E} \sum_{i=1}^{N}\left|\frac{\partial T_{k}\left(u_{n}\right)}{\partial x_{i}}\right|^{p} \omega_{i} d x .
$$

Owing to the strong compactness of $\left\{T_{k}\left(u_{n}\right)\right\}$ in $W_{0}^{1, p}(\Omega, \omega)$, there exists $\delta^{\prime}>0$ such that if meas $(E)<\delta^{\prime}$, then

$$
\int_{E \cap\left\{\left|u_{n}\right|<k\right\}} \sum_{i=1}^{N}\left|\frac{\partial u_{n}}{\partial x_{i}}\right|^{p} \omega_{i} d x \leq \frac{\varepsilon}{2}, \quad n \in N^{*}
$$


Hence,

$$
\int_{E} \sum_{i=1}^{N}\left|\frac{\partial u_{n}}{\partial x_{i}}\right|^{p} \omega_{i} d x \leq \varepsilon, \quad n \in N^{*}
$$

Thus, the sequence $\left\{\left|\nabla u_{n}\right|^{p}\right\}$ is equi-integrable. Thanks to Vitali theorem, the equi-integrability together with (76) implies that $u_{n}$ converges strongly to $u$ in $W_{0}^{1, p}(\Omega, \omega)$.

Step 4 (the strong convergence $g_{n}\left(x, u_{n}, \nabla u_{n}\right) \rightarrow g(x, u, \nabla u)$ in $L^{1}(\Omega)$ ). Note that (76) implies that

$$
g_{n}\left(x, u_{n}, \nabla u_{n}\right) \longrightarrow g(x, u, \nabla u) \text { a.e. in } \Omega .
$$

On the other hand, for any measurable set $E$ of $\Omega$, we have

$$
\begin{aligned}
& \int_{E}\left|g_{n}\left(x, u_{n}, \nabla u_{n}\right)\right| d x \\
& =\int_{E \cap\left\{\left|u_{n}\right|<k\right\}}\left|g_{n}\left(x, u_{n}, \nabla u_{n}\right)\right| d x \\
& \quad+\int_{E \cap\left\{\left|u_{n}\right| \geq k\right\}}\left|g_{n}\left(x, u_{n}, \nabla u_{n}\right)\right| d x .
\end{aligned}
$$

Let $\varepsilon>0$ be fixed. We have

$$
\begin{aligned}
& \int_{E \cap\left\{\left|u_{n}\right| \geq k\right\}}\left|g_{n}\left(x, u_{n}, \nabla u_{n}\right)\right| d x \\
& \leq \int_{\left\{\left|u_{n}\right| \geq k\right\}}\left|g_{n}\left(x, u_{n}, \nabla u_{n}\right)\right| d x .
\end{aligned}
$$

Choose $k \geq h$ in (46) such that

$$
\int_{E \cap\left\{\left|u_{n}\right| \geq k\right\}}\left|g_{n}\left(x, u_{n}, \nabla u_{n}\right)\right| d x \leq \frac{\varepsilon}{2}, \quad n \in N^{*} .
$$

By (10), we have

$$
\begin{aligned}
& \int_{E \cap\left\{\left|u_{n}\right|<k\right\}}\left|g_{n}\left(x, T_{k}\left(u_{n}\right), \nabla T_{k}\left(u_{n}\right)\right)\right| d x \\
& \leq b(k) \int_{E}\left|\frac{\partial T_{k}\left(u_{n}\right)}{\partial x_{i}}\right|^{p} \omega_{i}(x) d x+d(x) .
\end{aligned}
$$

Since $d(x)$ belongs to $L^{1}(\Omega)$ and $T_{k}\left(u_{n}\right)$ is compact in $W_{0}^{1, p}(\Omega, \omega)$, there exists $\delta^{\prime \prime}>0$ such that if meas $(E)<\delta^{\prime \prime}$, then

$$
\begin{aligned}
& \int_{E \cap\left\{\left|u_{n}\right|<k\right\}}\left|g_{n}\left(x, T_{k}\left(u_{n}\right), \nabla T_{k}\left(u_{n}\right)\right)\right| d x \leq \frac{\varepsilon}{2}, \\
& n \in N^{*} \text {. }
\end{aligned}
$$

Thus, we have proved that $\left\{g_{n}\left(x, u_{n}, \nabla u_{n}\right)\right\}$ is equi-integrable. Invoking (86) and (88) and by Vitali theorem,

$$
g_{n}\left(x, u_{n}, \nabla u_{n}\right) \longrightarrow g(x, u, \nabla u) \quad \text { strongly in } L^{1}(\Omega) .
$$

Step 5 (passing to the limit). Now, by passing to the limit in (27), we obtain

$$
\begin{gathered}
\int_{\Omega} a(x, u, \nabla u) \cdot \nabla v d x+\int_{\Omega} g(x, u, \nabla u) v d x \\
=\int_{\Omega} f v d x+\int_{\Omega} F \cdot \nabla v d x
\end{gathered}
$$

that is, $u$ is a weak solution to problem (1). The proof is complete.

\section{Conflict of Interests}

The authors declare that they have no competing interests.

\section{Authors' Contribution}

All authors contributed equally to the paper and read and approved its final version.

\section{Acknowledgment}

This study was supported by the National Science Foundation of China $(11271154,11401252)$.

\section{References}

[1] P. Drábek, A. Kufner, and V. Mustonen, "Pseudo-monotonicity and degenerated or singular elliptic operators," Bulletin of the Australian Mathematical Society, vol. 58, no. 2, pp. 213-221, 1998.

[2] Y. Akdim, E. Azroul, and A. Benkirane, "Existence of solutions for quasilinear degenerate elliptic equations," Electronic Journal of Differential Equations, vol. 2001, no. 71, pp. 1-19, 2001.

[3] K. Ammar, "Renormalized solutions of degenerate elliptic problems," Journal of Differential Equations, vol. 234, no. 1, pp. $1-25,2007$.

[4] L. Aharouch, E. Azroul, and A. Benkirane, "Quasilinear degenerated equations with $L^{1}$ datum and without coercivity in perturbation terms," Electronic Journal of Qualitative Theory of Differential Equations, vol. 19, article 18, 2006.

[5] V. Gol'dshtein and A. Ukhlov, "Weighted Sobolev spaces and embedding theorems," Transactions of the American Mathematical Society, vol. 361, no. 7, pp. 3829-3850, 2009.

[6] L. Boccardo, T. Gallouët, and L. Orsina, "Existence and nonexistence of solutions for some nonlinear elliptic equations," Journal d'Analyse Mathématique, vol. 73, pp. 203-223, 1997.

[7] Z. Li and W. Gao, "Existence of renormalized solutions to a nonlinear parabolic equation in $L^{1}$ setting with nonstandard growth condition and gradient term," Mathematical Methods in the Applied Sciences, vol. 38, pp. 3043-3062, 2015.

[8] A. Bensoussan, L. Boccardo, and F. Murat, "On a non linear partial differential equation having natural growth terms and unbounded solution," Annales de l'Institut Henri Poincaré (C) Analyse Non Linéaire, vol. 5, no. 4, pp. 347-364, 1988.

[9] L. Boccardo, A. Dall'Aglio, and L. Orsina, "Existence and regularity results for some elliptic equations with degenerate coercivity," in Atti del Seminario Matematico e Fisico dell'Università di Modena, vol. 46, pp. 51-81, Seminario Matematico e fisico Università di Modena, Dedicated to: C. Vinti (Perugia, 1996), 1998 (Italian). 
[10] H. Brézis and W. A. Strauss, "Semi-linear second-order elliptic equations in $L^{1}$," Journal of the Mathematical Society of Japan, vol. 25 , pp. 565-590, 1973. 


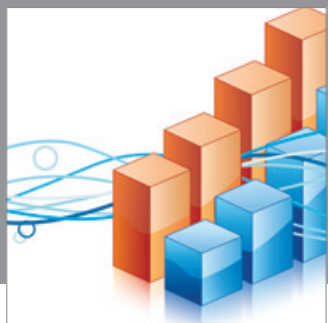

Advances in

Operations Research

mansans

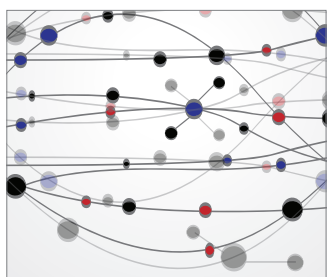

The Scientific World Journal
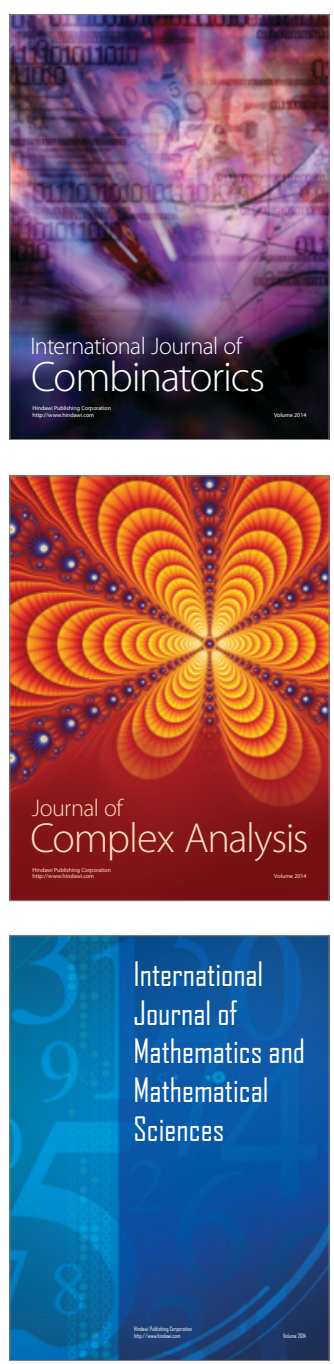
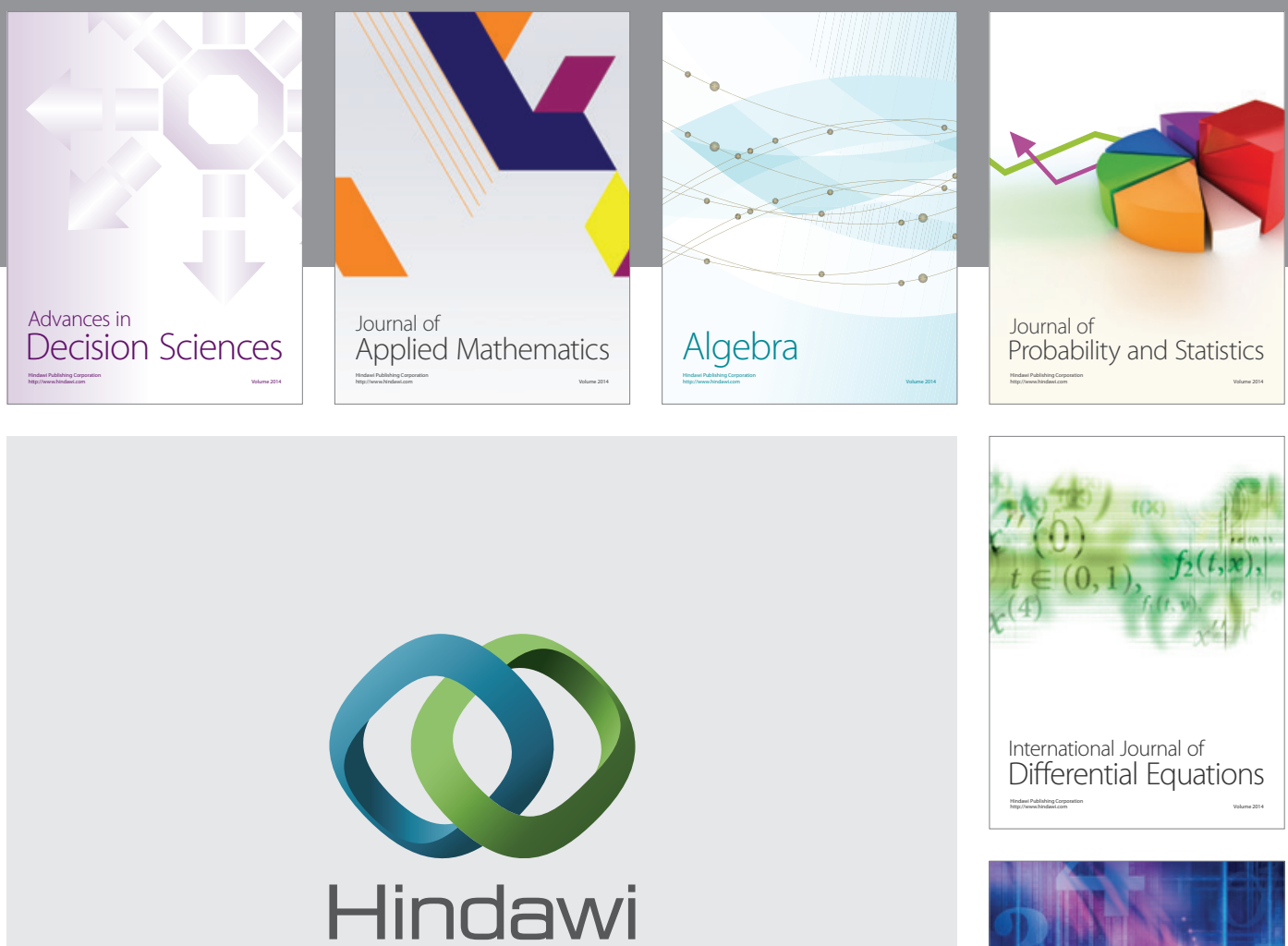

Submit your manuscripts at http://www.hindawi.com
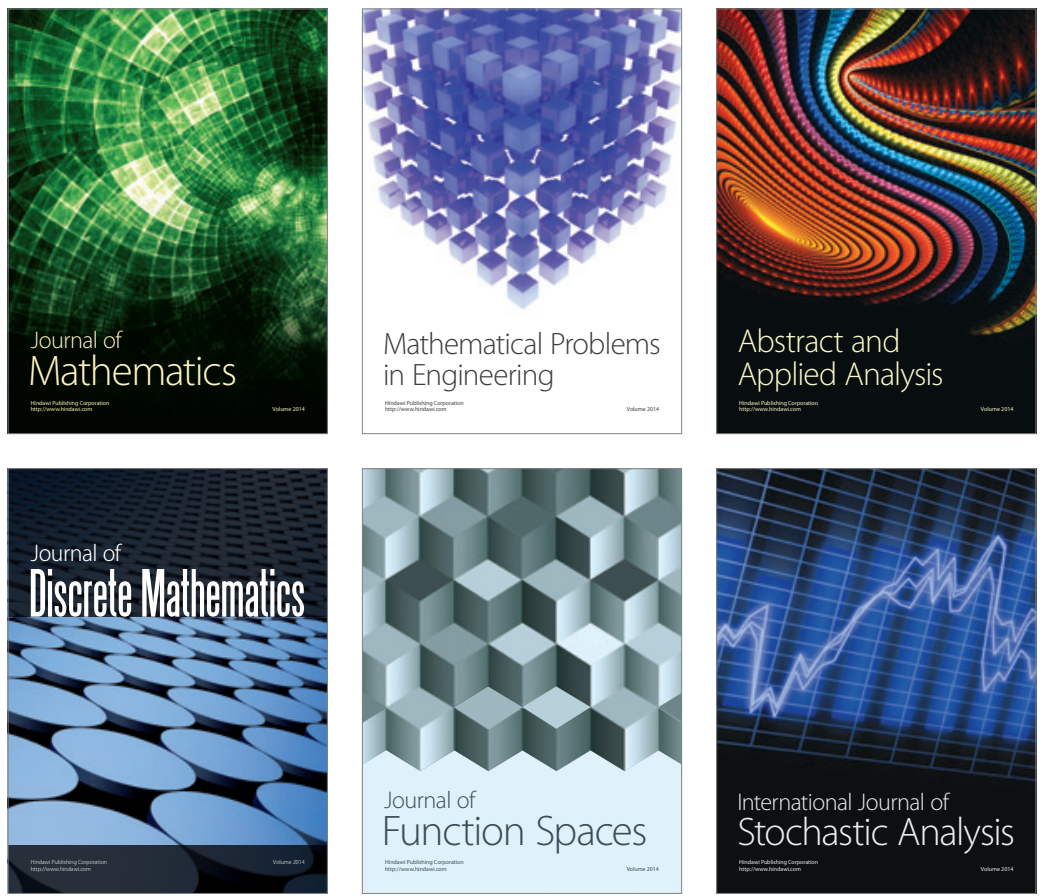

Journal of

Function Spaces

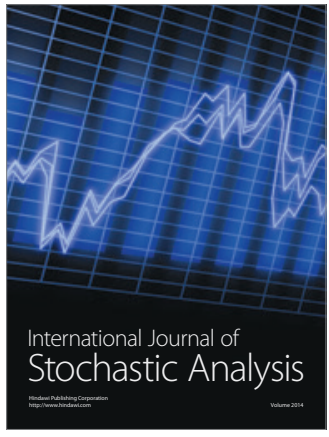

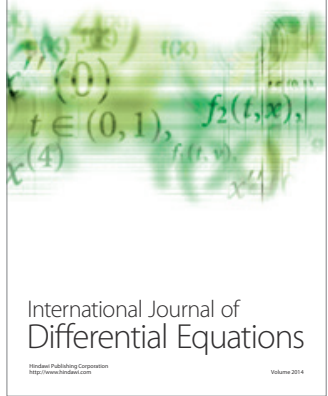
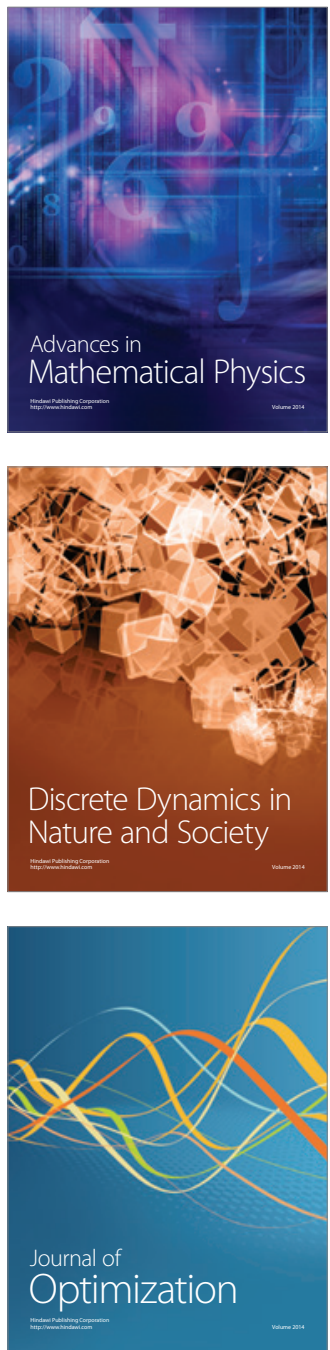